\title{
EDITORIAL
}

\section{Thoughts for a new year}

\author{
As we begin 2013 it's time to take stock of what we do know and what we don't, and consider \\ how best to approach finding new treatments for cancer. Do we embrace complexity or go \\ back to basics?
}

( biological insight is key together with data generation
Well, that's another year done and dusted, with not much time to pause for thought as we race into 2013. Thank you to everyone who wrote and refereed for us in 2012 and thank you in advance to the authors who have agreed to write in 2013.

So, what will a new year bring? More of the same perhaps? More 'omics'? More data? More questions and fewer answers? It's hard to see, even from our broad perspective, where cancer research is heading at the moment. Research into cancer has undoubtedly generated a lot of data and a lot of papers, but do enough of these papers provide insight? We now have a long, some would say 'laundry', list of genetic alterations that occur in different types of cancer. We have new and emerging techniques for developing nanotargeted drugs, new imaging approaches for diagnosis and prognosis, small interfering RNAs and new biological drugs that might find their way into the clinic, as well as mouse models that more closely mimic human disease. We have embraced the need for 'bench to bedside' and back again and are becoming able to plan ahead in terms of probable resistance mechanisms to targeted drugs. We now appreciate that cancers are more than just ever more mutated cells; we know they involve substantial interplay between cancer cells and their microenvironment and that, in addition to genetic changes, alterations that occur in non-coding RNAs and epigenetic regulators also have a considerable impact. Systemic effects, such as obesity and metabolic disease, probably impinge on cancer development and progression, and bringing the immune system into play for effective cancer treatment is likely to be important. We have new 'hallmarks' of cancer to play with, and we now know an awful lot compared with just 10 years ago, but deciding how best to use all of this knowledge in terms of treating cancer, especially metastatic cancer, is not always easy or clear. Are we now in danger of drowning in the data that we have generated from the 'omics' era? Although these data should be put to good use in the future, figuring out how to extract meaningful conclusions requires careful consideration, not least because cancer is such a complex disease.

Heterogeneity, or cancer plasticity, made bold headlines in 2012 for good reasons. Such findings suggest that each cancer patient really does present with a unique disease and that this uniqueness extends to the varieties of tumour cell clones that exist within an individual tumour ${ }^{1}$. Such results clearly indicate the levels of complexity surrounding tumour development, progression and response to treatment. As a recent Nature Outlook article ('Physical scientists take on cancer') ${ }^{2}$ expounds, cancer researchers are enlisting the help of their physical science colleagues to help tackle this complexity. Some are opting to work with scientists who understand the difficulties of handling huge amounts of data in order to extract a meaningful signal from the noise, while others are advocating that biologists need to propose more theories and to test these to help them to better understand cancer biology - akin to proposing the existence of the Higgs boson and developing the experimental means to prove or refute this. Others are using mathematical models to improve their comprehension of how tumours react to specific treatments. However, building mathematical and computer models to better understand cancer is limited by our understanding of cancer biology. So, do we need to go back to first principles? In one of the Nature Outlook articles Robert Gatenby argues, as he has done before in the pages of this journal ${ }^{3}$, that we need to understand how cancers evolve and the selective pressures that drive their evolution. In other words, that biological insight is key together with data generation.

In thinking about cancer complexity and helping others to understand the intricacies that surround it, we need to be mindful of the language that we use. In particular, we should avoid falling into the trap of describing cancer in anthropomorphic terms, just because this is an easier way to convey our ideas. Implying, for example, that cancer cells knowingly plan a route to the brain, lung or bone by writing that a cancer or cancer cell selects or keeps a specific mutation or increased expression of a microRNA glosses over a whole heap of multifaceted biology and is of course factually inaccurate. So, along with drinking less alcohol and going to the gym more often, our New Year's resolutions could include avoiding writing about cancer as a disease with a 'plan' and finding another way to simplify the intricacies of cancer.

\footnotetext{
. Gerlinger, M. et al. N. Engl. J. Med. 366, 883-892 (2012).

2. Gravitz, L. Nature 491, S49 (2012).

. Gatenby, R. A., Gillies, R. J. \& Brown, J. S. Nature Rev. Cancer 11, 237-238 (2011)
} 